\title{
When different norms lead to same billiard trajectories?
}

\author{
Arseniy Akopyan ${ }^{1} \cdot$ Roman Karasev ${ }^{2,3}$
}

Received: 1 January 2020 / Accepted: 21 March 2020 / Published online: 15 April 2020

(c) The Author(s) 2020

\begin{abstract}
Extending a result of Milena Radnović and Serge Tabachnikov, we establish conditions for two different non-symmetric norms to define the same billiard reflection law.
\end{abstract}

Keywords Billiards · Finsler geometry · Hamiltonian systems

Mathematics Subject Classification 53B40 - 53D99 $\cdot$ 70H05

Radnović [6] and independently Tabchnikov [7, Section 2] made the following remarkable observation:

Theorem 1 Let $\|\cdot\|_{\xi}$ be a not necessarily symmetric norm in the plane, having an ellipse with focus at the origin o as the unit circle. Then $\|\cdot\|_{\xi}$ defines the same law of reflection as the Euclidean metric: The angle of reflection equals to the angle of incidence.

In the first paper it was also noticed that it leads to the fact that billiard trajectories in the plane with norm defined by an ellipse as the unit circle, are the same as in the Euclidean plane after a suitably chosen affine transform.

\begin{abstract}
AA was supported by European Research Council (ERC) under the European Union's Horizon 2020 research and innovation programme (Grant Agreement No. 78818 Alpha). RK was supported by the Federal professorship program Grant 1.456.2016/1.4 and the Russian Foundation for Basic Research Grants 18-01-00036 and 19-01-00169.
\end{abstract}

$凶$ Arseniy Akopyan

akopjan@gmail.com

Roman Karasev

r_n_karasev@mail.ru

1 Institute of Science and Technology Austria (IST Austria), Am Campus 1, 3400 Klosterneuburg, Austria

2 Moscow Institute of Physics and Technology, Institutskiy per. 9, Dolgoprudny, Russia 141700

3 Institute for Information Transmission Problems RAS, Bolshoy Karetny per. 19, Moscow, Russia 127994 


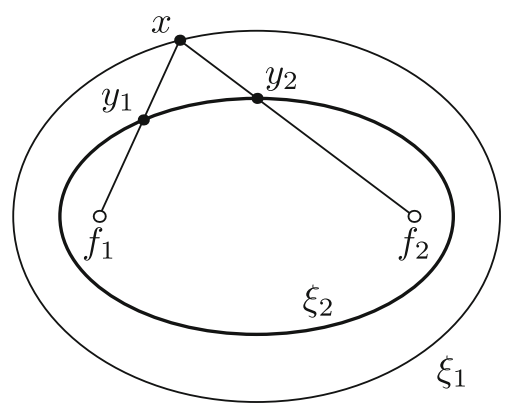

Fig. $1 \frac{\left|x f_{1}\right|}{\left|y_{1} f_{1}\right|}+\frac{\left|x f_{2}\right|}{\left|y_{2} f_{2}\right|}=$ const

Another consequence of this theorem is that the Euclidean and normed ellipses with foci $o$ and $f$ coincide, where $f$ is the second focus of $\xi$. Indeed, if $x$ is a point in the plane, then by Theorem 1 the differential of $\|\overrightarrow{o x}\|_{\xi}+\|\overrightarrow{x f}\|_{\xi}$ is proportional to the differential of the same expression for the Euclidean norm for every $x$. Hence the value $\|\overrightarrow{o x}\|_{\xi}+\|\overrightarrow{x f}\|_{\xi}$ does not change when $x$ moves along the ellipse with foci $f$ and $o$, in the zero direction of both differentials.

It is interesting, that the latter statement may be deciphered to the following elementary geometric formulation, for which we do not know any short synthetic proof essentially different from the one stated in the above paragraph.

Corollary 2 Let $\xi_{1}$ and $\xi_{2}$ be two confocal ellipses with foci at $f_{1}$ and $f_{2}$. For each point $x$ on $\xi_{1}$, denote by $y_{1}$ and $y_{2}$ the points of intersections of $\xi_{2}$ with rays $f_{1} x$ and $f_{2} x$ respectively (Fig. 1). Then for any point $x$ on $\xi_{1}$,

$$
\frac{\left|x f_{1}\right|}{\left|y_{1} f_{1}\right|}+\frac{\left|x f_{2}\right|}{\left|y_{2} f_{2}\right|}=\text { const. }
$$

It can be shown, that the constant in the corollary above equals

$$
\frac{\ell_{1}-\left|f_{1} f_{2}\right|}{\ell_{2}-\left|f_{1} f_{2}\right|}+\frac{\ell_{1}+\left|f_{1} f_{2}\right|}{\ell_{2}+\left|f_{1} f_{2}\right|}
$$

where $\ell_{1}$ and $\ell_{2}$ are the major axes of the ellipses $\xi_{1}$ and $\xi_{2}$.

Now we extend the Radnović-Tabachnikov theorem to normed spaces in higher dimension.

Theorem 3 Let $K$ be a smooth convex body in $\mathbb{R}^{n}$ containing the origin, and $T$ be its convex image under a projective transform, that maps each line passing through origin to itself preserving its orientation at the origin (Fig. 2). Then the billiard reflection law in the space with norm $\|\cdot\|_{K}$ is the same as in the space with norm $\|\cdot\|_{T}$.

Remark 4 It is known (see [3, Lemma 4.6]) that such kind of projective transforms send spheres with center at origin to ellipsoids with one of the foci at the origin. Therefore this theorem directly generalizes Theorem 1 to higher dimension. 


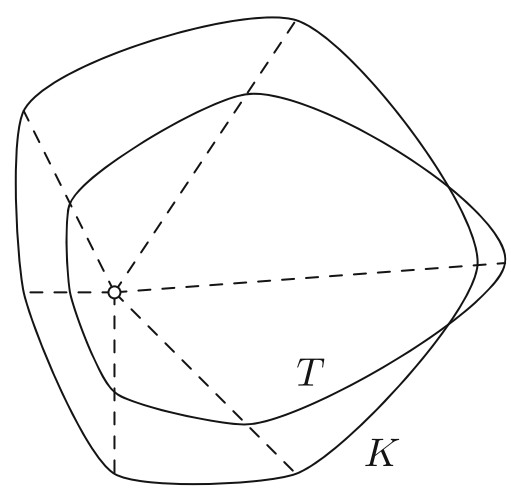

Fig. 2 Convex body $K$ and its projective image $T$

Remark 5 The law of reflection is not well defined for convex bodies $K$ that are not strictly convex. In this case we may follow the conventions in [1] and define billiard trajectories, for which the reflection direction is not uniquely defined.

Proof We use an idea from [2,4,5], suggesting to work with billiard trajectory in the Banach space $U=\mathbb{R}^{n}$ with norm $\|\cdot\|_{K}$ in terms of momenta in the dual space $U^{*}$ with norm $\|\cdot\|_{K^{\circ}}$, whose unit ball is the polar body $K^{\circ}$. From the smoothness of $K$, to each unit velocity $u \in \partial K$ there corresponds a conjugate unit momentum $u^{*} \in \partial K^{\circ}$ such that $u^{*}(u)=1$ and $\left\|u^{*}\right\|_{K^{\circ}}=1$. The equation $u^{*}(x)=1$ defines the hyperplane tangent to $\partial K$ at $u$, while the equation $u(y)=1$ defines the hyperplane tangent to $\partial K^{\circ}$ at $u^{*}$.

Let $q_{1} q_{2} q_{3}$ be a part of a billiard trajectory in $U$, where $q_{2}$ is the point where the trajectory hits a hypersurface $S$ and reflects. Then the sum $\left\|\overrightarrow{q_{1} x}\right\|_{K}+\left\|\overrightarrow{x q_{3}}\right\|_{K}$ as a function of $x \in S$ has a critical value at $q_{2}$. The criticality in terms of first derivatives means:

$$
u_{2}^{*}-u_{1}^{*}=\lambda n^{*}
$$

where $u_{1}^{*}$ and $u_{2}^{*}$ are momenta corresponding to unit vectors in directions $\overrightarrow{q_{1} q_{2}}$ and $\overrightarrow{q_{2} q_{3}}$, and $n^{*}$ is the normal covector to $S$ at $q_{2}$.

It is crucial that equation $(*)$ is preserved under a positive similarity of the body $K^{\circ}$, possibly with different factor $\lambda$. Indeed, let $T^{\circ}=t K^{\circ}+v^{*}$, where $t>0$ and $v^{*} \in U^{*}$. It is easy to see that the momentum, corresponding to velocity $u$ with respect to the body $T^{\circ}$, equals $u_{T}^{*}=t u^{*}+v^{*}$, because $u$ is a linear function on $U^{*}$ and the points, where its maximum is obtained on $K^{\circ}$ and $T^{\circ}$ are moved one to another by the homothety. Hence the difference of the new momenta at a reflection point equals to $t\left(u_{2}^{*}-u_{1}^{*}\right)$, which is still parallel to $n^{*}$.

We obtain that the reflection laws for two norms $\|\cdot\|_{K}$ and $\|\cdot\|_{T}$ coincide if $K^{\circ}$ and $T^{\circ}$ are positive homothets of each other. A positive homothety is a projective transform which maps any point at infinity to itself. Therefore in the dual space (our original $U$ ) a positive homothety corresponds to the map, which preserves its polar 
images as sets, that is planes passing through the origin. It is easy to see that this is the projective transform described in the statement of the theorem.

In simpler words, $K$ is given by the system of linear inequalities of the form

$$
u^{*}(x) \leqslant 1, \text { for all } u^{*} \in K^{\circ} .
$$

Hence the equations of $T$ must be (assuming working not far from the origin, where $v(x)<1)$

$$
t u^{*}(x)+v^{*}(x) \leqslant 1 \Longleftrightarrow u^{*}\left(\frac{t x}{1-v(x)}\right) \leqslant 1, \quad \text { for all } u^{*} \in K^{\circ}
$$

It remains to note that

$$
x \mapsto \frac{t x}{1-v(x)}, \quad t>0,
$$

is the general form of projective maps that preserve lines thorough the origin and keep their orientations at the origin.

Acknowledgements Open access funding provided by Institute of Science and Technology (IST Austria). The authors thank Alexey Balitskiy, Milena Radnović, and Serge Tabachnikov for useful discussions.

Open Access This article is licensed under a Creative Commons Attribution 4.0 International License, which permits use, sharing, adaptation, distribution and reproduction in any medium or format, as long as you give appropriate credit to the original author(s) and the source, provide a link to the Creative Commons licence, and indicate if changes were made. The images or other third party material in this article are included in the article's Creative Commons licence, unless indicated otherwise in a credit line to the material. If material is not included in the article's Creative Commons licence and your intended use is not permitted by statutory regulation or exceeds the permitted use, you will need to obtain permission directly from the copyright holder. To view a copy of this licence, visit http://creativecommons.org/licenses/by/4.0/.

\section{References}

1. Akopyan, A., Balitskiy, A.: Billiards in convex bodies with acute angles. Israel J. Math. 216(2), 833-845 (2016)

2. Akopyan, A., Balitskiy, A., Karasev, R., Sharipova, A.: Elementary approach to closed billiard trajectories in asymmetric normed spaces. Proc. Amer. Math. Soc. 144(10), 4501-4513 (2016)

3. Akopyan, A.V., Bobenko, A.I.: Incircular nets and confocal conics. Trans. Amer. Math. Soc. 370(4), 2825-2854 (2018)

4. Artstein-Avidan, S., Ostrover, Y.: A Brunn-Minkowski inequality for symplectic capacities of convex domains. Int. Math. Res. Not. IMRN 2008, \# rnn044 (2008)

5. Gutkin, E., Tabachnikov, S.: Billiards in Finsler and Minkowski geometries. J. Geom. Phys. 40(3-4), 277-301 (2002)

6. Radnović, M.: A note on billiard systems in Finsler plane with elliptic indicatrices. Publ. Inst. Math. (Beograd) (N.S.) 74(88), 97-101 (2003)

7. Tabachnikov, S.: Remarks on magnetic flows and magnetic billiards, Finsler metrics and a magnetic analog of Hilbert's fourth problem. In: Brin, M., Hasselblatt, B., Pesin, Y. (eds.) Modern Dynamical Systems and Applications, pp. 233-250. Cambridge University Press, Cambridge (2004)

Publisher's Note Springer Nature remains neutral with regard to jurisdictional claims in published maps and institutional affiliations. 